\title{
Research on the Sustainable Development of Yoga Teaching in Colleges and Universities
}

\author{
Youfeng Wang ${ }^{1}$ \\ ${ }^{1}$ Department of Physical Education of Oxbridge College, Kunming University of Science and \\ Technology, Kunming, Yunnan Province, China
}

\section{Keywords: Colleges And Universities; Yoga Teaching; Sustainable Development; Countermeasures.}

\begin{abstract}
At present, many students are keen to learn yoga, not only for fitness, but also for the fashion in universities and colleges. For the physical education in colleges, in order to meet the needs of the university students, yoga is a health project, which has been welcomed by university students. Especially the girls in universities are keen to participate in yoga activities. Through the observation and analysis of the students participating in yoga, it is found that the students who participate in yoga training have obvious improvement in their physical and mental health. However, there are still some problems in the process of yoga teaching for the university students because of the lack of in-depth study on the teaching of yoga. In order to promote the sustainable development of yoga teaching in universities and colleges, it is necessary to study the relevant problems. This paper studies the sustainable development of yoga teaching in universities and colleges.
\end{abstract}

\section{Introduction}

Yoga is a sport that is originated from India. In recent years, China is becoming more and more popular, especially for women who are very keen on yoga. Yoga combines dance, gymnastics, Tai Chi, as well as the concept of traditional Chinese medicine. The studies of yoga involve a large number of disciplines. First of all, yoga is a sports category with a high artistic value, as well as philosophy. Yoga has become a fashionable sport in our society [1]. Among the university students, most of them love yoga. In some universities and colleges, they offer yoga teaching, which can not only deepen the students' physical education, but also help to promote the students' healthy development. Yoga is very realistic. As a form of sports, yoga is a combination of sports and art. The students who participate in the yoga exercise can not only play the role of physical exercise, but also help to improve their artistic aesthetic level. When the students receive yoga education, they can not only be in accordance with the guidance of teachers, but also accept the relevant culture, which will ease themselves in yoga education and help them to release their mental pleasure [2]. Therefore, yoga can promote the students' physical and mental health.

\section{Research Participants}

The participants of this study are selected from Kunming University of Science and Technology, Yunnan University of Nationalities, Yunnan Normal University. The four hundred students involved in yoga are all girls entering universities in 2015 and 2016.

\section{Research Methods}

In this study, we use the methods of questionnaire survey and follow-up investigation. The method of questionnaire adopts the method of issuing questionnaires. The follow-up investigation adopts the method of face-to-face communication and telephone communication. All questionnaires were distributed to the girls who participate in yoga. 400 pieces of questionnaires were issued and 396 pieces of questionnaires were effective. The follow-up investigation mainly adopts face-to-face communication. In their spare time, the researchers communicate with the students through close communication. During the communication, the researchers gained the students' ideas of yoga and 
the improvement of yoga. During the follow-up communication within two semesters since the participants start to study yoga, the researchers conducted a survey on the girls' personality, explored the girls' development and made a summary.

\section{Research Results and Analysis}

The students' interest changes after participating in yoga. In the development of the university students' personality, their interest is very important for their growth. Different university students have different hobbies, which will be reflected after they participate in yoga. The students who choose to take part in yoga will be very interested in the sports. These students who want to participate in yoga can improve their physical and mental health. Therefore, the university students who participate in yoga to meet their needs. Each student's interest in yoga is also different, that is, the degree of preference for yoga is determined by their subjective consciousness is different. They participate in yoga with different purposes. Through the use of questionnaires, the teachers can clearly understand the students' ideas and have in-depth understanding of the students' needs of yoga education. During the survey, 30\% of the students think that they like yoga and they participate in it. Yoga activities can be organized by the school, which is very popular. However, they are not able to meet the students' needs. Although these students hold different views on the teaching of yoga, they do not want to give up the sport of yoga. The interest of the students in this group is relatively stable. $36 \%$ of the students believe that they are very fond of yoga. However, most of them cannot continue to participate in the sport for a long time because of many factors. The main reason is that the students' personal condition and schoolwork make them participate in other sports. Moreover, yoga education may beyond the students' imagination. Therefore, the students will adhere to yoga in a period of time from the perspective of trying and they may give up and adhere to other sports. $19 \%$ of the students do not have the perceptual knowledge of yoga. The results of the questionnaire do not show their interest of yoga. They do not have clear attitude to yoga. 15\% of the students decide to give up yoga and choose other sports items. It mainly because that after receiving yoga education, the students think that they feel uncomfortable and gave up it.

From the survey data, it is clear that the students' interest in yoga is in an unstable state. They only have the perceptual knowledge of yoga instead of understanding it. In addition, they will be affected by a variety of factors and attitudes towards yoga [3]. When the university students are interested in yoga, the yoga education is difficult to meet their needs and they will choose to give up the sport and to participate in other sports until they feel satisfactory.

The students' personality changes in yoga. Among the university students, if the female groups have enough time and energy, they will choose to participate in yoga, not only for fitness, but also for public meeting. The students can exchange with each other for a wide range of topics. Yoga culture is one aspect of the topics. It can also help the students to achieve the purpose of fitness. However, many universities and colleges female girls participate in yoga exercise to become healthy. In order to improve the teaching effect of yoga, the teachers will require the students to participate in yoga. They should start from the students' subjective interest. If they love this operation, they can have a clear purpose in participating in sports, which will ensure that the students will not give up yoga and they can persist without being influenced by many factors. For the students participating in yoga, they think that their shaping is correlated to their employment. In order to improve their image and make themselves more confident and positive, the college girls participate in yoga. Thus, their interest is only one aspect while they have strong purpose, which is bound to the personality of the university students. Yoga has a guiding significance in the development of the university students' personality. Through yoga, the teachers can adjust their body and minds, help to promote the students' physical and mental development. By participating in yoga, the students can not only create a beautiful body, but also can have a more positive attitude and improve their aesthetic ability [4]. In the melodious rhythm of music, the university students do yoga to cultivate their sentiment, become healthy and create a good artistic temperament. Yoga is in accordance with the rhythm of the music movement, so that it can help the students to develop their flexibility and coordination and promote the body's growth and development. For the university students, in the employment competition, a good image 
has been the first impression. It is very suitable to choose their own career. For the students who are going to graduate from school, they prefer to choose the ideal occupation. Figure and appearance is the first impression, which is the basic condition to improve their self-confidence [5]. After learning some simple actions, the students can follow the teachers' instructions and act in the accompaniment of music. Yoga music is also a very dynamic choice of music. When the students hear the music, it will arouse their feeling of action and it will be enthusiastic to participate in yoga. It can be seen that the yoga has certain influence on the students' mental health.

According to the results of the questionnaire survey, 396 pieces of the questionnaires were effective in the study. 105 pieces were from Northeastern University, accounting for $26.51 \%$ of the total questionnaires; 183 pieces were from Liaoning University, accounting for $46.21 \%$ of the total questionnaires; 108 pieces were from Shenyang University of Technology, accounting for $27.27 \%$ of the total questionnaires. From the perspective of the development of the students' personality, these students are not mature enough both physically and psychologically. These require further improvement [6]. These university students have better vision of their own future. They focus on their basis vision which is not based on the fantasy elements which are higher. If not given proper guidance, it is easy to lose a sense of loss and melancholy ideal. For vibrant and energetic university students, they have directions and they make efforts. Thus, they have a sunny life which is full of strong sense of pursuit. The students were mostly born after 1990. They love new things, love to show their love and explore the mystery. However, they also have distinctive personality. They mainly participate in the yoga because of their interest. After a period of time in which they receive yoga education, the students will have a preliminary understanding of yoga, have more specific yoga training motivation, have higher requirements to improve their physique and try to give people better visual sense of beauty, which is helpful to improve their occupation competitiveness, improve their self-confidence in front of their classmates [7]. In the process of taking part in yoga, it also helps to improve the students' health, establish their aesthetic consciousness, improve their aesthetic level, so that their personality can be effectively demonstrated. Therefore, the university students who participate in yoga actually reflect their personality.

Through the questionnaire survey, more than $90 \%$ of the students have passionate attitude to yoga. They love sports because yoga is melodious with slow music. The meditation training of yoga and the various graceful actions of yoga will make the university students participate in yoga. The rhythm of the music will make the students be more willing to release themselves through yoga. This method can help the teachers and students to communicate and solve their troubles. At the same time, they can also display the personality, help the students change their behaviors, promote their initiative and make themselves feel well in sports. From the perspective of psychology, when the students participate in yoga, they will have varying degrees of psychological changes. These changes will be shown in their actions [8]. The yoga teachers can understand the students' ideas and solve their problems through the students' actions. They can also guide the students to let your heart close down, have more imagination, remove distractions and fully ease themselves. While the students devote themselves to yoga, they will gradually improve their enthusiasm for yoga. The higher students' enthusiasm will contribute to the students' more interest and better learning effectiveness. They will be more willing to show themselves in this way. It can be seen that yoga has great influence on the development of the students' personality.

\section{Conclusions}

To sum up, the physical education in universities and colleges should be synchronized with the society, so that the students can keep up with the pace of the social development and they will not fall behind in the trend of the times. In the social competition, people have rapid pace of life, as well as large pressure. Therefore, most people have been in a state of sub-health. This kind of social phenomenon also has a deep influence on the university students. They are about to enter the society. The unhealthy physical and mental health will inevitably affect the quality of their work. The introduction of yoga in higher education can promote the development of the students' physical and mental health and it is helpful to cultivate the students' habit of participating in physical exercise. 
Yoga is graceful and it can help to shaping, which can guide the students to master their self-cultivation. It is very necessary for yoga teaching in universities and colleges to maintain the sustainable development.

\section{References}

[1] Hai-yuan Liu \& Deng-song Zhou. Discussion on Guiding Ideology in Physical Education Teaching and its Basic Train of Thought [J]. Beijing: JOURNAL OF BEIJING UNIVERSITY OF PHYSICAL EDUCATION, 2013 (04): 86-88.

[2] Li Zhao. On the Evolution and Development of School Physical Education Thought and Practice in China [J]. SPORT SCIENCE, 2014 (05): 77-78.

[3] Zhi-ying Wei. Reserach on the Present Condition of Yoga Teaching in Colleges and Universities of Hunan Province [J]. JOURNAL OF HUNAN INSTITUTE OF HUMANITIES SCIENCE AND TECHNOLOGY, 2015(03): 85-87.

[4] Wu Yang. On the Health-oriented Guiding Ideology of School [J]. CHINA SCIENCE AND TECHNOLOGY INFORMATION, 2015 (10): 75-76.

[5] Li Jiang. A Study on the Development Strategy of Sports and the Future Development Trend of Sports in China [J]. Journal of Chengdu Sport University, 2015 (01): 96-97.

[6] Di Zhang. Reflections on the Teaching of Yoga in Universities and Colleges [J]. Education Art, 2016 (06): 69-70.

[7] Xiao-ling Luo. Discussion on the Factors and Countermeasures of Sustainable Development of Physical Education in Universities and Colleges [J]. HIGHER EDUCATION IN CHEMICAL ENGINEERING, 2014 (01): 16-17.

[8] Shao-zeng Liu. Learning to Understand and Implement: Earnestly Implement the Health-oriented Guiding Ideology [J]. Physical Education, 2014 (03): 44-45. 